\title{
Gastrointestinal tract spindle cell tumors with interstitial cells of Cajal: Prevalence excluding gastrointestinal stromal tumors
}

\author{
SO JUNG LEE ${ }^{1}$, CHUNG SU HWANG ${ }^{2}$, AHRONG KIM $^{1}$, KYUNGBIN KIM $^{3 *}$ and KYUNG UN CHOI ${ }^{1,4^{*}}$ \\ ${ }^{1}$ Department of Pathology, Pusan National University Hospital, Busan 49241; ${ }^{2}$ Department of Pathology, \\ Pusan National University Yangsan Hospital, Yangsan, Gyeongsangnam 50612; ${ }^{3}$ Department of Pathology, \\ Ulsan University Hospital, Ulsan 44033; ${ }^{4}$ Department of Pathology, School of Medicine, \\ Pusan National University, Yangsan, Gyeongsangnam 50612, Republic of Korea
}

Received February 3, 2016; Accepted April 18, 2016

DOI: $10.3892 / \mathrm{ol} .2016 .4758$

\begin{abstract}
Leiomyomas and schwannomas of the gastrointestinal tract (GIT) are mainly comprised of spindle-shaped tumor cells and should always be differentiated from gastrointestinal stromal tumors (GISTs). Mast/ stem cell growth factor receptor Kit (KIT) and discovered on GIST-1 (DOG1) are well-known diagnostic markers for the detection of a GIST by immunohistochemical staining. The aim of the present study was to assess the prevalence and significance of spindle cell tumors of the GIT with KIT- or DOG1-positive spindle-shaped cells, presumed to be interstitial cells of Cajal (ICCs), other than GISTs. A total of 71 leiomyomas and 35 schwannomas were examined and clinicopathological information was obtained.KIT and DOG1 immunostaining was performed to determine the proportions of positive cells. Mutation screening of KIT exons 9, 11, 13 and 17, and platelet-derived growth factor receptor $\alpha$ (PDGFRA) exons 12 and 18 was performed in cases with a relatively high proportion of either KIT- or DOG1-positive cells. The frequency of leiomyomas and schwannomas with KIT- and DOG1-positive ICCs was $35.2 \%$ (25/71 cases) and 5.7\% (2/35 cases), respectively. Among the esophageal leiomyomas with KIT- and DOG-positive ICCs (14/25; 56.0\%), 5 leiomyomas involved the muscularis mucosa and 9 leiomyomas involved the muscularis propria. All gastric leiomyomas with KIT- and DOG1-positive ICCs (11/25; 44\%)
\end{abstract}

Correspondence to: Dr Kyungbin Kim, Department of Pathology, Ulsan University Hospital, 877 Bangeojinsunhwando-Ro, Dong, Ulsan 44033, Republic of Korea

E-mail: cinema7@hanmail.net

Dr Kyung Un Choi, Department of Pathology, School of Medicine, Pusan National University, 49 Busandaehak-Ro, Mulgeum-eup, Yangsan, Gyeongsangnam 50612, Republic of Korea

E-mail: kuchoi@pusan.ac.kr

*Contributed equally

Key words: leiomyoma, schwannoma, gastrointestinal stromal tumor, KIT, DOG1, interstitial cells of Cajal involved the muscularis propria. All schwannomas with an increased proportion of KIT- or DOG1-positive ICCs were of gastric origin. No KIT or PDGFRA mutations were detected in 7 leiomyomas and 2 schwannomas. In conclusion, the majority of leiomyomas and the minority of schwannomas in the GIT had a significant portion of KIT- and DOG1-positive cells. All of the tumors were located in the upper GIT, and could be present in the muscularis propria or muscularis mucosa. The tumors represented a non-neoplastic proliferation of KIT- and DOG1-positive cells in the GIT. Careful evaluation of KIT- or DOG1-positive cells in spindle cell tumors of the GIT can assist in forming the correct diagnosis by differentiation from a GIST.

\section{Introduction}

Mesenchymal tumors of the gastrointestinal tract (GIT) are mainly comprised of a spectrum of spindle cell tumors, including gastrointestinal stromal tumors (GISTs), leiomyomas, schwannomas, inflammatory fibroid polyps, fibromatoses, and more rarely, leiomyosarcomas. GISTs should always be considered in the differential diagnosis of spindle cell tumors of the GIT, as GISTs constitute the largest subset of mesenchymal tumors of the GIT and GIST cell morphology is usually spindle-shaped $(70 \%)$, although certain GISTs consist of epithelioid cells $(20 \%)$ or a mixture of cells $(1,2)$. GISTs may be defined as mesenchymal tumors of the GIT that usually express the mast/stem cell growth factor receptor Kit (KIT) protein and harbor mutations of a gene that encodes for KIT or platelet-derived growth factor receptor $\alpha$ (PDGFRA) and probably originate from the interstitial cells of Cajal (ICCs) (3). Since KIT and PDGFRA tyrosine kinase inhibitors have become available, the proper identification of GISTs has become clinically important $(4,5)$. Therefore, it is necessary to differentiate other mesenchymal tumors of the GIT from GISTs, particularly those consisting of spindle-shaped tumor cells.

KIT and DOG1 immunohistochemical staining is a well-known diagnostic tool for detecting GISTs $(6,7)$. Notably, certain lesions among the mesenchymal tumors of the GIT, other than GISTs, are represented by a substantial number of KIT-positive or DOG1-positive spindle-shaped cells, presumed 
to be interstitial cells of Cajal (ICCs). If this finding is obtained in a small biopsy, tumors with a high proportion of KIT- or DOG1-positive spindle-shaped cells can mimic a GIST.

The present study assessed the incidence and significance of KIT- or DOG1-positive spindle-shaped cells in leiomyoma or schwannoma of the GIT, which is the main differential diagnosis of spindle cell tumors of the GIT.

\section{Materials and methods}

Patients and tissue samples. Samples of the spindle cell tumors of the GIT, coded as leiomyoma or schwannoma, were obtained by polypectomy and endoscopic or surgical resection performed at Pusan National University Yangsan Hospital (Yangsan, Gyeongsangnam, South Korea) or Pusan National University Hospital (Busan, South Korea) between 2009 and 2014. A total of 106 patients (mean age, 32 years; range, $18-84$ years) were examined based on the availability of material and clinicopathological information. The present study was approved by the Institutional Review Board at Pusan National University Yangsan Hospital after obtaining written informed consent.

Clinicopathological data, including age, gender, tumor type, tumor size and tumor location, were recorded. Follow-up information was also recorded. The results of previously evaluated immunohistochemical staining for smooth muscle actin (SMA), desmin and S100 protein were obtained from pathology reports. Hematoxylin and eosin-stained slides were reviewed, and previously performed immunohistochemical staining for SMA, desmin and S100 protein was reassessed. Table I provides an overview of the main clinicopathological features.

Immunohistochemistry. In all 106 cases, additional KIT and DOG1 immunostaining was performed to determine the proportions of positive cells within leiomyomas or schwannomas of the GIT. Rabbit polyclonal anti-human CD117/c-KIT antibody (1:600 dilution; Dako, Carpinteria, CA, USA) and rabbit monoclonal anti-DOG1 (SP31) antibody (1:500 dilution; Cell Marque, Rocklin, CA, USA) were used for immunostaining. Immunohistochemistry was performed on serial 4- $\mu \mathrm{m}$ thick, formalin-fixed paraffin-embedded (FFPE) blocks using the EnVision Detection system (Dako) with diaminobenzidine as the chromogen, according to the manufacturer's protocols.

Immunohistochemical staining was interpreted by two independent pathologists. KIT and DOG1 immunostaining was assessed over the range of $0-100 \%$ positive staining of cells. Staining of the sections with antibodies against KIT and DOG1 was considered significant when $>5 \%$ of the cells within the tumor were positive.

Analysis of c-Kit and PDGFRA gene mutations. Mutation screening of KIT exons 9,11, 13 and 17, and PDGFRA exons 12 and 18 was performed in selective cases with a relatively high proportion of either KIT- or DOG1-positive cells (>15\%).

DNA was extracted from five $10-\mu \mathrm{m}$ thick FFPE sections containing a representative portion of tumor tissue using the QIAamp DNA FFPE Tissue kit (Qiagen, Hilden, Germany) according to the manufacturer's protocols. DNA (50 ng) was amplified in a $20-\mu 1$ reaction solution containing $10 \mu$ l of
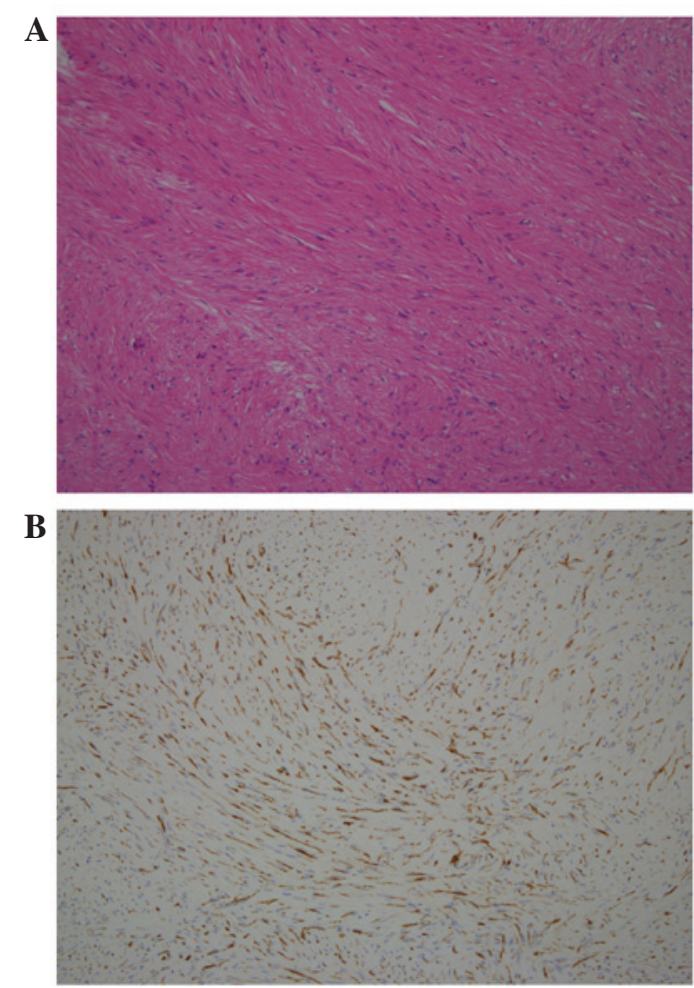

Figure 1. Leiomyoma. (A) Leiomyoma composed of intersecting fascicles of spindle-shaped smooth cells with eosinophilic cytoplasm (hematoxylin and eosin staining; magnification, x100). (B) An immunohistochemical stain for DOG-1 showing a high density of DOG1-positive interstitial cells of Cajal within the tumor (magnification, x200). DOG1, discovered on GIST-1.

2X concentrated HotStarTaq Master mix (Qiagen), including polymerase chain reaction (PCR) buffer with $3 \mathrm{mM} \mathrm{MgCl}_{2}$, $400 \mu \mathrm{M}$ of each dNTP and $0.3 \mu \mathrm{M}$ of each primer pair. PCR products were gel purified on $2 \%$ gels with a QIAgen gel extraction kit (Qiagen). DNA templates were processed for the DNA sequencing reaction with forward and reverse sequence-specific primers using the ABI-PRISM BigDye Terminator ver. 3.1 (Applied Biosystems; Thermo Fisher Scientific, Waltham, MA, USA). Sequence data were generated with the ABI PRISM 3100 DNA Analyzer, and sequences were analyzed by Sequencing analysis 5.1.1. software (both Applied Biosystems; Thermo Fisher Scientific Inc.) to compare variations.

\section{Results}

The study population was comprised of 71 cases of leiomyoma and 35 cases of schwannoma. The age and gender distribution of the 106 cases with pathological data (tumor site, location, size and results for SMA, desmin and S100 protein) is shown in Table I. Leiomyomas were more common in the esophagus and schwannomas were more frequent in the stomach. The majority of leiomyomas and schwannomas originated from the muscularis propria. Leiomyomas measured $0.2-11.0 \mathrm{~cm}$ in their greatest dimension (median, $1.9 \mathrm{~cm}$ ), and schwannomas measured 0.5-8.0 cm (median, $2.5 \mathrm{~cm}$ ). All masses included in this study were well circumscribed and were composed of spindle cells that did not show nuclear atypia or pleomorphism. Mitoses were absent or few in number. Leiomyomas (Fig.1) 
Table I. Clinicopathological characteristics of the spindle cell tumors of the gastrointestinal tract.

\begin{tabular}{|c|c|c|}
\hline Characteristic & Leiomyoma $(\mathrm{n}=71)$ & Schwannoma $(n=35)$ \\
\hline Mean age, years & 34 & 27 \\
\hline Gender ratio (male:female) & $37: 34$ & $13: 23$ \\
\hline Mean tumor size, $\mathrm{cm}$ & 2.6 & 3.0 \\
\hline \multicolumn{3}{|l|}{ Tumor distribution } \\
\hline \multicolumn{3}{|l|}{ Esophagus } \\
\hline MM & 15 & 0 \\
\hline MP & 20 & 2 \\
\hline \multicolumn{3}{|l|}{ Stomach } \\
\hline MM & 3 & 2 \\
\hline MP & 18 & 25 \\
\hline \multicolumn{3}{|l|}{ Small intestine } \\
\hline $\mathrm{MM}$ & 0 & 0 \\
\hline MP & 2 & 0 \\
\hline \multicolumn{3}{|l|}{ Colon and rectum } \\
\hline MM & 10 & 3 \\
\hline MP & 3 & 3 \\
\hline \multicolumn{3}{|l|}{ Immunohistochemistry } \\
\hline SMA & All diffusely-positive & All negative \\
\hline Desmin & All diffusely-positive & All negative \\
\hline S-100 protein & All negative & All diffusely-positive \\
\hline
\end{tabular}

MM, muscularis mucosa; MP, muscularis propria.

Table II. Spindle cell tumors of the gastrointestinal tract with mast/stem cell growth factor receptor Kit- and discovered on GIST-1-positive interstitial cells of Cajal.

\begin{tabular}{lcc}
\hline Characteristics & $\begin{array}{c}\text { Leiomyoma } \\
(\mathrm{n}=25)\end{array}$ & $\begin{array}{c}\text { Schwannoma } \\
(\mathrm{n}=2)\end{array}$ \\
\hline Mean age, years & 49.5 & 62 \\
Gender ratio (male:female) & $13: 12$ & $0: 2$ \\
Mean tumor size, cm & 3.1 & 2.8 \\
Tumor distribution & & \\
Esophagus & & 0 \\
$\quad$ MM & 5 & 0 \\
MP & 9 & 0 \\
Stomach & & 2 \\
MM & 0 & \\
MP & 11 & \\
\hline
\end{tabular}

MM, muscularis mucosa; MP, muscularis propria.

showed intersecting fascicles of spindled cells that closely resembled normal smooth muscle cells with eosinophilic cytoplasm (Fig. 1A) and schwannomas (Fig. 2) were composed of spindled cells with elongated, wavy nuclei (Fig. 2A). Leiomyomas were diffusely-positive for SMA and desmin, and negative for S100 protein, whereas all schwannomas showed
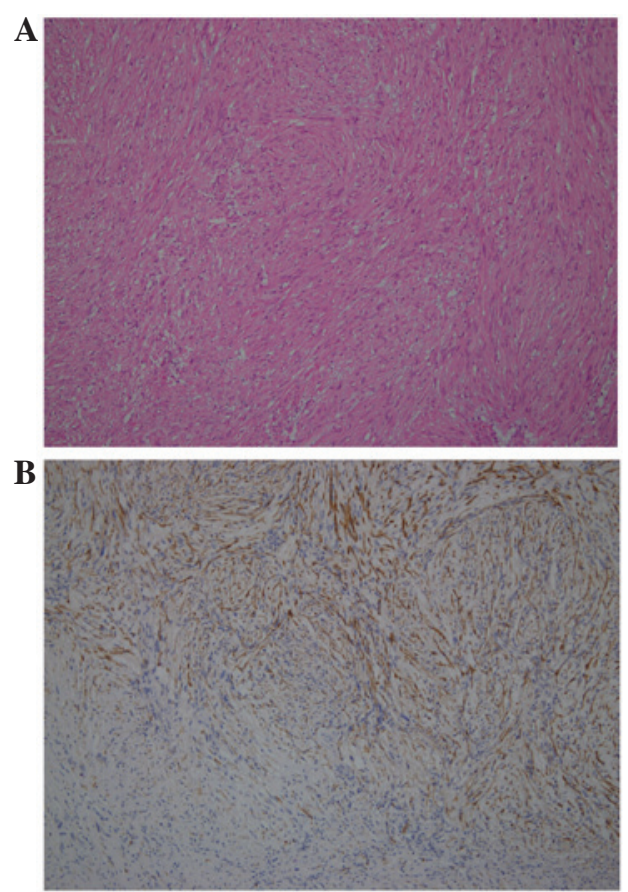

Figure 2. Schwannoma. (A) Schwannoma composed of short bundles of spindle-shaped Schwann cells (hematoxylin and eosin staining; magnification, x100). (B) In a few cases, the number of discovered on GIST-1-positive interstitial cells of Cajal were significantly increased (magnification, x200).

completely opposite patterns (data not shown). None of the patients were reported as experiencing tumor recurrence. 
KIT immunoreactivity was usually diffuse and cytoplasmic, but occasionally it showed a membranous or a dot-like pattern, and DOG1 staining appeared predominately localized to the plasma membrane (Figs. 1B and 2B). Mast cells were identified in all KIT and DOG1 immunohistochemically-stained slides. In addition to the mast cells, the slides showed a second population of KIT- or DOG1-positive cells. These cells were easily differentiated from mast cells based on their spindle shape and long dendritic processes typical of ICCs. In general, these cells were diffusely or sparsely distributed throughout the tumors, but focal aggregates were identified in certain cases. There was no significant ICC hyperplasia in the adjacent muscle layers in all cases.

The proportion of KIT- and DOG1-positive ICCs varied in leiomyomas from 0 to $30 \%$ of the lesional cells. The frequency of leiomyomas with a significant proportion of KIT- and DOG1-positive ICCs was 35.2\% (25/71 cases) (Fig. 1B). Of these 25 cases, $14(56.0 \%)$ were located in the esophagus and $11(44.0 \%)$ were located in the stomach. Among the esophageal leiomyomas with KIT- and DOG1-positive ICCs (14/35, $40.0 \%), 5$ leiomyomas involved the muscularis mucosa and 9 leiomyomas involved the muscularis propria. All gastric leiomyomas with KIT- and DOG1-positive ICCs $(11 / 21$, $52.4 \%$ ) involved the muscularis propria. By contrast, few KIT- or DOG1-positive ICCs or no expression was detected in the majority of schwannomas, with the exception of 2 cases in which 15 and $20 \%$ of the lesional cells were KIT- and DOG1-positive ICCs, respectively (Fig. 2B). All these tumors were gastric shwannomas arising from the muscularis propria (Table II).

Mutation analysis of KIT exons 9, 11, 13 and 17, and PDGFRA exons 12 and 18 was performed in the 7 leiomyomas and 2 schwannomas that exhibited $>15 \%$ KIT- and DOG1-positive lesional cells. No KIT or PDGFRA mutations were detected in any of these 9 cases (Fig. 3).

\section{Discussion}

The present study examined the incidence and distribution of KIT- or DOG1-positive cells in spindle cell tumors of the GIT, and assessed their true nature by performing KIT and PDGFRA mutation analysis to rule out the possibility of a GIST. Leiomyomas and schwannomas of the GIT mainly exhibit spindle cell morphology and should always be differentiated from a GIST. KIT- and DOG1-positive cells in spindle cell tumors of the GIT represent ICCs, as these cells closely resemble normal ICCs with their dendritic-like processes and are reactive to KIT and DOG1, which are robust ICC markers (8). The present study found that these cells were present in gastric schwannomas $(8 \%)$, as well as in esophageal $(40 \%)$ and gastric $(52.4 \%)$ leiomyomas, particularly in the muscularis propria. The cells are diffusely distributed or focally aggregated.

Several studies have noted KIT- or DOG1-positive cells in leiomyomas of the GIT, which were interpreted as a colonization of Cajal cells between smooth muscle cells $(4,8,9)$. Deshpande et al (9) reported that a significant number of upper deep leiomyomas of the GIT possessed KIT- or DOG1-positive ICCs, and an increase in the numbers of KIT- or DOG1-positive ICCs within a tumor raised doubt as to whether the mass was a GIST. Paying careful attention to the morphological appearance of these cells, as well as the tumor cells, can assist in establishing the differential diagnosis of a GIST, particularly in a small biopsy due to the clinical relevance. The present study also observed that KIT- or DOG1-positive ICCs were absent in the lower GIT. However, the study showed that leiomyomas that arose from the musularis mucosa also possessed a significant proportion of KIT- or DOG1-positive ICCs, particularly in the esophagus $(5 / 15,33.3 \%)$. KIT- or DOG1-positive cells could be distinguished by their long and slender dendritic-like processes eminating from elongated and plump smooth cells with blunt-ended nuclei and an eosinophilic cytoplasm.

By contrast, 2 cases of gastric schwannomas in the GIT exhibited KIT- or DOG1-positive ICCs and each possessed a significant proportion of these cells. Previous studies have reported the absence of KIT-positive cells in schwannomas of the GIT $(10,11)$. Schwannomas of the GIT exhibit morphological clues that can aid in making a diagnosis, such as wavy Schwann cells with a tapered end and occasionally prominent lymphoid cuffs in the periphery (10), even if there is an area with a high density of KIT- or DOG1-positive cells, particularly in resected specimens. However, we suggest that gastric schwannoma should be considered if a spindle cell tumor is detected on a small biopsy from a gastric lesion when a gastrointestinal mesenchymal tumor is clinically suspected.

Several hypotheses have been described for explaining the presence of ICCs: i) Infiltration and hyperplasia by non-neoplastic ICCs from the adjacent muscularis propria, in which KIT- or DOG1-positive ICCs are normally detected; and ii) a type of stromal tumor with mixed differentiation, including ICCs, smooth muscle and neural elements (8). In the present study, it was found that the distribution of KIT- or DOG1-positive ICCs was occasionally diffuse throughout leiomyomas and schwannomas and not prominent at the peripheral portion of the tumors. There was no significant ICC hyperplasia in the adjacent tissue in all cases. Furthermore, the absence of KIT and PDGFRA mutations in cases of tumors with a significant proportion of KIT- and DOG1-positive ICCs supports the notion that these cells were not true neoplastic GIST cells, but that they were non-neoplastic ICCs between tumor cells.

In conclusion, the majority of leiomyomas and the minority of schwannomas in the GIT have up to $30 \%$ of all lesional cells formed from KIT- and DOG1-positive cells, respectively. KIT- and DOG1-positive cells have dendritic-like processes closely resembling normal ICCs and are reactive to the markers of ICCs, namely, KIT and DOG1. All of the tumors were located in the upper GIT, and moreover, all schwannomas with increased proportions of KIT- or DOG1-positive ICCs were of gastric origin. The tumors could be present in the muscularis propria or muscularis mucosa. Furthermore, KIT and PDGFRA mutations were absent in these tumors. It was concluded that these tumors represent a non-neoplastic proliferation of KIT- and DOG1-positive cells in the GIT. The presence of a large numbers of these cells prompts the diagnosis of a GIST, particularly in limited biopsy samples. Careful evaluation of KIT- or DOG1-positive cells in spindle cell tumors of the GIT that are located in the muscularis proper or mucosa can 
A

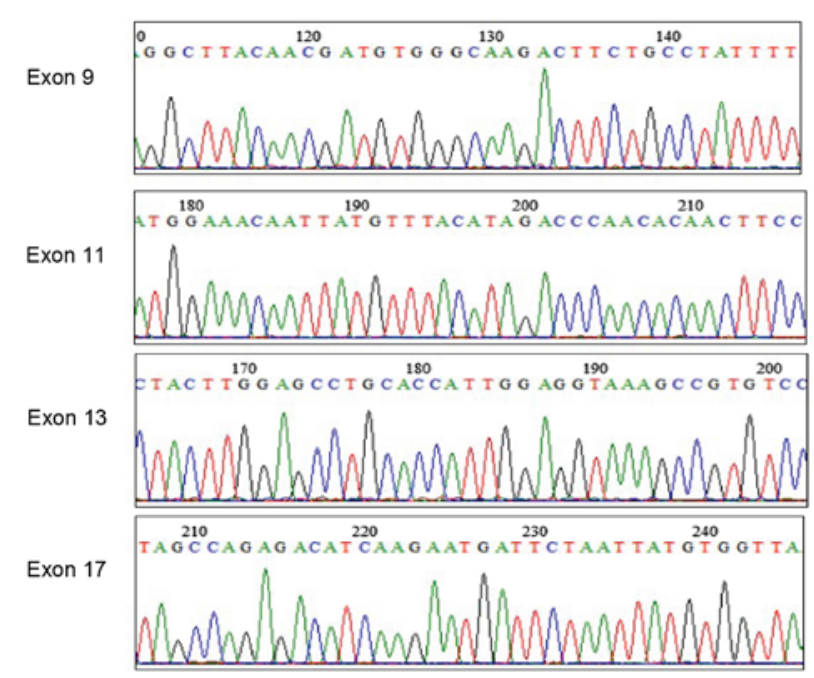

B

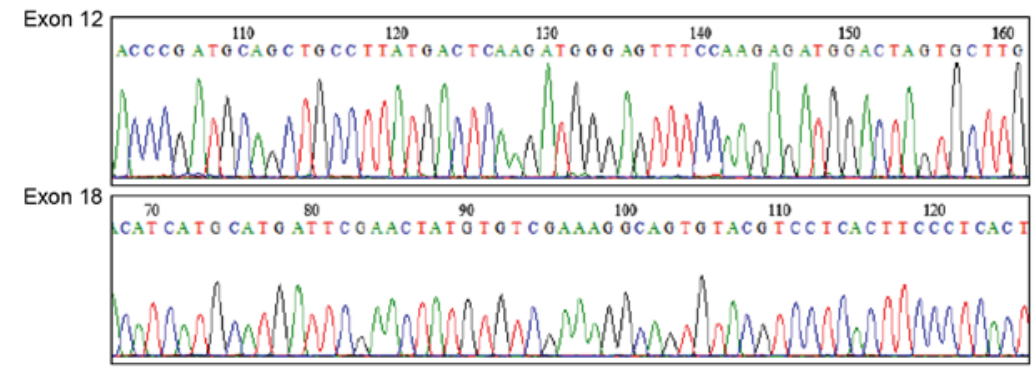

C
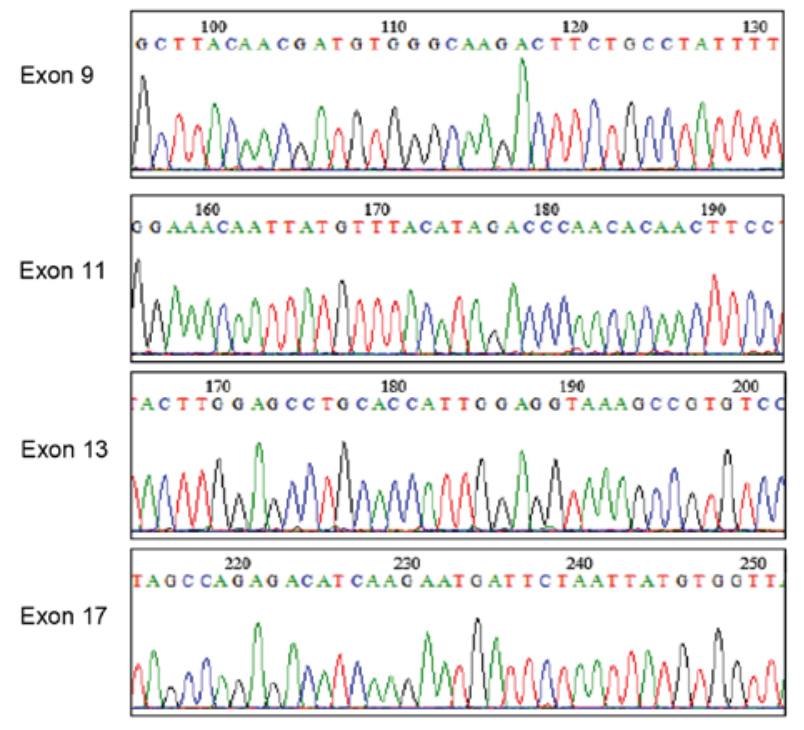

D

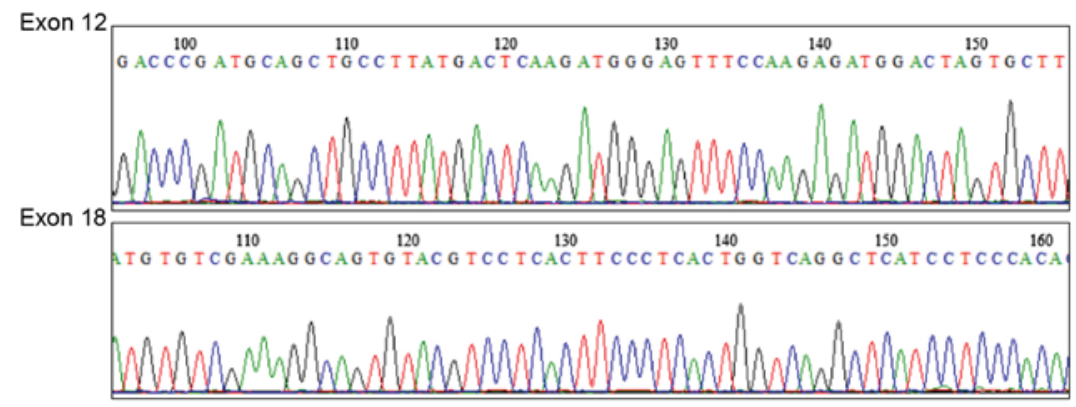

Figure 3. KIT and PDGFRA mutation analyses on representative cases. (A) KIT exon 9, 11, 13 and 17 mutations are absent in leiomyoma. (B) PDGFRA exon 12 and 18 mutations are absent in leiomyoma. (C) KIT exon 9, 11, 13, and 17 mutations are absent in schwannoma. (D) PDGFRA exon 12 and 18 mutations are absent in schwannoma. KIT, mast/stem cell growth factor receptor Kit; DOG1, discovered on GIST-1; PDGFRA, platelet-derived growth factor receptor $\alpha$. 
aid in making the correct diagnosis by differentiating the tumors from a GIST.

\section{Acknowledgements}

This study was supported by a grant from the National R\&D Program for Cancer Control, the Ministry for Health, and the Welfare and Family Affairs, Republic of Korea (no. 0920050).

\section{References}

1. Miettinen M, Sarlomo-Rikala M and Sobin LH: Mesenchymal tumors of muscularis mucosae of colon and rectum are benign leiomyomas that should be separated from gastrointestinal stromal tumors - A clinicopathologic and immunohistochemical study of eighty-eight cases. Mod Pathol 14: 950-956, 2001.

2. Rosai J: Gastrointestinal stromal tumor and its mimics. Int J Surg Pathol 18 (3 Suppl): S79-S87, 2010.

3. Kindblom LG, Remotti HE, Aldenborg F and Meis-Kindblom JM: Gastrointestinal pacemaker cell tumor (GIPACT): Gastrointestinal stromal tumors show phenotypic characteristics of the interstitial cells of Cajal. Am J Pathol 152: 1259-1269, 1998.

4. Miettinen M, Wang ZF and Lasota J: DOG1 antibody in the differential diagnosis of gastrointestinal stromal tumors: A study of 1840 cases. Am J Surg Pathol 33: 1401-1408, 2009.
5. Miettinen M, Sobin LH and Lasota J: Gastrointestinal stromal tumors of the stomach: A clinicopathologic, immunohistochemical, and molecular genetic study of 1765 cases with long-term follow-up. Am J Surg Pathol 29: 52-68, 2005.

6. Miettinen M and Lasota J: Gastrointestinal stromal tumors-definition, clinical, histological, immunohistochemical, and molecular genetic features and differential diagnosis. Virchows Arch 438: 1-12, 2001.

7. West RB, Corless CL, Chen X, Rubin BP, Subramanian S, Montgomery K, Zhu S, Ball CA, Nielsen TO, Patel R, et al: The novel marker, DOG1, is expressed ubiquitously in gastrointestinal stromal tumors irrespective of KIT or PDGFRA mutation status. Am J Pathol 165: 107-113, 2004

8. Kim JM, Kim A, Choi JH and Bae YK: Usefulness of DOG1 expression in the diagnosis of gastrointestinal stromal tumors. Korean J Pathol 44: 141-148, 2010.

9. Deshpande A, Nelson D, Corless CL, Deshpande V and O'Brien MJ. Leiomyoma of the gastrointestinal tract with interstitial cells of Cajal: A mimic of gastrointestinal stromal tumor. Am J Surg Pathol 38: 72-77, 2014.

10. Miettinen M, Sarlomo-Rikala M, Sobin LH and Lasota J: Esophageal stromal tumors: A clinicopathologic, immunohistochemical, and molecular genetic study of 17 cases and comparison with esophageal leiomyomas and leiomyosarcomas. Am J Surg Pathol 24: 211-222, 2000.

11. Hou YY, Tan YS, Xu JF, Wang XN, Lu SH, Ji Y, Wang J and Zhu XZ: Schwannoma of the gastrointestinal tract: A clinicopathological, immunohistochemical and ultrastructural study of 33 cases. Histopathology 48: 536-545, 2006. 